\title{
Heavy Thermal Dark Matter from a New Collision Mechanism
}

\author{
Eric David Kramer@* and Eric Kuflik® $\oplus^{\dagger}$ \\ Racah Institute of Physics, Hebrew University of Jerusalem, Jerusalem 91904, Israel \\ Noam Levi ${ }^{\ddagger}$ and Nadav Joseph Outmezguine $\odot^{\S}$ \\ School of Physics and Astronomy, Tel-Aviv University, Tel-Aviv 69978, Israel \\ Joshua T. Ruderman $\|$ \\ Center for Cosmology and Particle Physics, Department of Physics, New York University, New York, New York 10003, USA
}

(Received 13 May 2020; revised 2 December 2020; accepted 2 February 2021; published 22 February 2021)

\begin{abstract}
We propose a new thermal freeze-out mechanism that results in dark matter masses exceeding the unitarity bound by many orders of magnitude, without violating perturbative unitarity or modifying the standard cosmology. The process determining the relic abundance is $\chi \zeta^{\dagger} \rightarrow \zeta \zeta$, where $\chi$ is the dark matter candidate. For $m_{\zeta}<m_{\chi}<3 m_{\zeta}, \chi$ is cosmologically long-lived and scatters against the exponentially more abundant $\zeta$. Therefore, such a process allows for exponentially heavier dark matter for the same interaction strength as a particle undergoing ordinary $2 \rightarrow 2$ freeze-out, or equivalently, exponentially weaker interactions for the same mass. We demonstrate this mechanism in a leptophilic dark matter model, which allows for dark matter masses up to $10^{9} \mathrm{GeV}$.
\end{abstract}

DOI: 10.1103/PhysRevLett.126.081802

Introduction.-The observational evidence for the existence and ubiquity of dark matter (DM) is well established, yet its origin and particle nature remain unknown. This puzzle has driven several decades of exploration into the landscape of potential DM models and cosmological mechanisms for producing the relic abundance of DM. Among these, the prospect of DM particles thermally coupled to the standard model (SM) in the early Universe has been especially prominent.

During the cosmological evolution of the Universe, the interaction rate between the SM and the dark sector is fast at early times, keeping the two baths in chemical and thermal equilibrium. When the interaction rate falls below the Hubble expansion rate, chemical equilibrium between the SM and the dark sector ceases. Soon afterward, the interactions completely stop and the abundance of DM is set by a process known as thermal freeze-out. The most widely explored paradigm representing this concept is the weakly interacting massive particle (WIMP), where the relic abundance is set by the freeze-out of DM-DM annihilations to the SM. The WIMP is a particularly promising candidate, as it predicts weak scale DM masses and annihilation rates, thus relating DM production to the new physics frontier.

Published by the American Physical Society under the terms of the Creative Commons Attribution 4.0 International license. Further distribution of this work must maintain attribution to the author(s) and the published article's title, journal citation, and DOI. Funded by SCOAP ${ }^{3}$.
Within the WIMP scenario, there is an upper bound on the mass of DM set by perturbative unitarity of roughly $m_{\text {WIMP }} \sim 100 \mathrm{TeV}$ [1]. Are there minimal extensions to the WIMP paradigm that predict heavier DM masses than this unitarity bound, leading to qualitatively different experimental signatures? Attempts at answering this question have focused on out-of-equilibrium dynamics with the SM and/or nonstandard cosmological histories [2-23]. If DM is a composite object, such as a hadron, the unitarity bound applies to the size of the object and not the masses of its constituents [1,24-28].

Thermal freeze-out mechanisms considering topologies beyond the WIMP have been considered [29-34], but until recently none have evaded the unitarity bound. A thermal mechanism that exceeds the unitarity bound, without modifying the standard cosmology, was proposed for the first time in Ref. [35], requiring a chain of interactions but allowing DM masses as high as $10^{14} \mathrm{GeV}$. In this Letter, we present a new thermal two-to-two freeze-out mechanism that requires just two interactions and allows for DM masses as heavy as $10^{10} \mathrm{GeV}$, without violating unitarity or modifying the standard cosmology. Conversely, this mechanism can achieve thermal weak scale DM, but with much smaller interaction rates than the WIMP.

Our setup consists of a DM candidate $(\chi)$, a SM portal, and at least one extra interacting degree of freedom in the

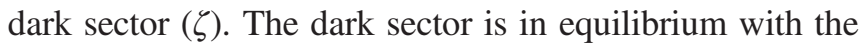
SM at early times, maintained by $\zeta \zeta^{\dagger}$ annihilations to the $\mathrm{SM}$, and internally in equilibrium via the process $\chi \zeta^{\dagger} \rightarrow \zeta \zeta$ between the two dark particles. When the masses of the 
dark sector particles obey the hierarchy $m_{\zeta}<m_{\chi}$, heavy DM naturally arises in our setup, as we detail below. The reason is that the mass splitting causes $\zeta$ to be exponentially more abundant than $\chi$ in chemical equilibrium, allowing for $\chi$ to be removed by scattering efficiently against a particle that is exponentially more abundant than itself. Note that a similar process was considered in Ref. [15] for heavy DM within a nonthermal and nonstandard cosmological history; in contrast, here we show how to obtain heavy DM thermally and within a standard cosmology.

General idea.-Consider a DM particle $\chi$ whose number density changes at early times via an interaction of the form $\mathcal{L} \supset \chi^{\dagger} \zeta \zeta \zeta$, with some field $\zeta$. The DM number density can deplete via the process $\chi \zeta^{\dagger} \rightarrow \zeta \zeta$. This process behaves similar to a zombie infection, where a $\zeta$ particle (the zombie), infects the DM $\chi$ and turns it into a zombie. The final DM abundance consists of the $\chi$ that persist after this process decouples. We refer to this process as a "zombie collision."

We restrict ourselves to $m_{\zeta}<m_{\chi}<3 m_{\zeta}$, where such interactions do not induce (on shell) $\chi \rightarrow \zeta \zeta \zeta$ decays, and the process $\chi \zeta^{\dagger} \rightarrow \zeta \zeta$ does not enter a forbidden regime [36,37]. Whenever $m_{\chi}>m_{\zeta}$, the process $\chi \zeta^{\dagger} \rightarrow \zeta \zeta$ can maintain chemical equilibrium longer than if the $\chi$ particle was annihilating with another $\chi$, because the interaction rate is proportional to $n_{\zeta}$, which is exponentially larger than $n_{\chi}$ when $\chi$ becomes nonrelativistic. Thus, if this process is responsible for $\chi$ freeze-out, the correct relic abundance is obtained for smaller interactions than WIMP-like DM, for the same DM mass. Similarly, since zombie collisions can be very efficient, this scenario also allows for heavier DM than the WIMP unitarity bound, without violating unitarity.

To realize this mechanism, we consider the two processes shown in Fig. 1, $\chi \zeta^{\dagger} \rightarrow \zeta \zeta$ and $\zeta \zeta^{\dagger} \rightarrow$ sm sm, where "sm" is a light particle which is either part of the SM bath or thermalized with it. The possibility of other interactions, such as $\chi \chi^{\dagger}$ annihilations, will be discussed below in the context of a specific UV model. Coscattering [33,35,38,39] is a different example in which the DM abundance can be set by the decoupling of two-to-two scattering of DM against a lighter state.

The Boltzmann equations governing the evolution of the $\chi$ and $\zeta$ number densities are given by
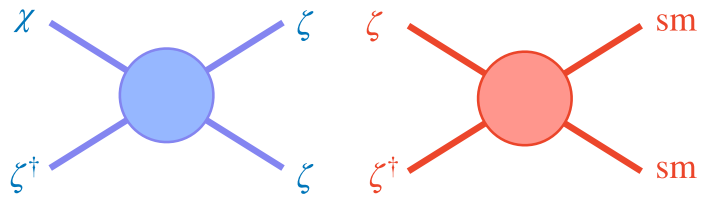

FIG. 1. Schematic representation of the processes setting the relic abundance. On the left, $\chi$ is our DM candidate and $\zeta$ is a hidden sector zombie particle that turns a $\chi$ into a $\zeta$. The process on the right maintains chemical equilibrium between the dark sector and the SM.

$$
\begin{gathered}
\dot{n}_{\chi}+3 H n_{\chi}=-\left\langle\sigma_{\chi \zeta \rightarrow \zeta \zeta} v\right\rangle\left(n_{\chi} n_{\zeta}-n_{\zeta}^{2} \frac{n_{\chi}^{\mathrm{eq}}}{n_{\zeta}^{\mathrm{eq}}}\right), \\
\dot{n}_{\zeta}+\dot{n}_{\chi}+3 H\left(n_{\zeta}+n_{\chi}\right)=-\left\langle\sigma_{\zeta \zeta \rightarrow \mathrm{sm} \mathrm{sm}} v\right\rangle\left(n_{\zeta}^{2}-n_{\zeta}^{\mathrm{eq} 2}\right),
\end{gathered}
$$

where the superscript "eq" denotes equilibrium abundances at zero chemical potential. The density of $\chi$ will depart from its equilibrium distribution (and freeze out soon after) when the rate of the $\chi$ number-changing process drops below the Hubble expansion rate. This happens approximately when [see the Supplemental Material [40] or Eq. (5.40) in [41] ]

$$
n_{\zeta}\left(x_{\chi}\right)\left\langle\sigma_{\chi \zeta \rightarrow \zeta \zeta} v\right\rangle=x_{\chi} H\left(x_{\chi}\right),
$$

where we have defined $x \equiv m_{\chi} / T$ and $x_{\chi}$ is the temperature when $\chi$ departs equilibrium. Equation (3) determines this temperature, which will be used to estimate the $\chi$ relic abundance. Unlike the WIMP, the freeze-out dynamics depends on whether the zombies $\zeta$ follow an equilibrium distribution or instead have already frozen out from the thermal bath by the time $\chi$ departs equilibrium with the $\zeta$. We describe these possibilities and their different phases below.

Equilibrium phase-zombies in equilibrium throughout freeze-out: When the $\zeta \zeta^{\dagger} \leftrightarrow$ sm sm interactions are efficient in maintaining equilibrium of $\zeta$ with the SM bath, $\chi$ evolves according to the Boltzmann equation

$$
\dot{n}_{\chi}+3 H n_{\chi}=-n_{\zeta}^{\mathrm{eq}}\left\langle\sigma_{\chi \zeta \rightarrow \zeta \zeta} v\right\rangle\left(n_{\chi}-n_{\chi}^{\mathrm{eq}}\right) .
$$

The relic abundance can be estimated using the instantaneous freeze-out approximation, utilizing the fact that, at freeze-out, the $\chi-\zeta$ system is still in equilibrium,

$$
n_{\chi}(x) \simeq r^{3 / 2} \exp \left[-\frac{r-1}{r} x\right] n_{\zeta}(x),
$$

where we defined $r \equiv m_{\chi} / m_{\zeta}>1$. Parametrizing the cross section as $\left\langle\sigma_{\chi \zeta \rightarrow \zeta \zeta} v\right\rangle \equiv \alpha_{\chi}^{2} / m_{\chi}^{2}$, the DM mass required to match the observed abundance is

$$
m_{\chi} \simeq\left[\left(\alpha_{\chi}^{2} m_{\mathrm{pl}}\right)^{r} T_{\mathrm{eq}}\right]^{\frac{1}{1+r}},
$$

where $T_{\text {eq }} \simeq 0.8 \mathrm{eV}$ is the temperature at matter radiation equality and $m_{\mathrm{pl}}$ is the Planck mass (see the Supplemental Material [40] for full derivation). The same expression with $r=1$ is the known relationship from the standard WIMP calculation. The gain in DM mass over the WIMP is evident from the equation above, showing an exponentially larger weight on $m_{\mathrm{pl}}$ vs $T_{\mathrm{eq}}$. The thermal evolution of this phase, for the specific model we describe later, is shown in the blue curves in Fig. 2.

In most models, one would expect $\chi \chi$ to also annihilate to the SM or zombies $\zeta$. If these annihilations are faster at freeze-out than $\chi \zeta^{\dagger} \rightarrow \zeta \zeta$, then the DM will behave as a 


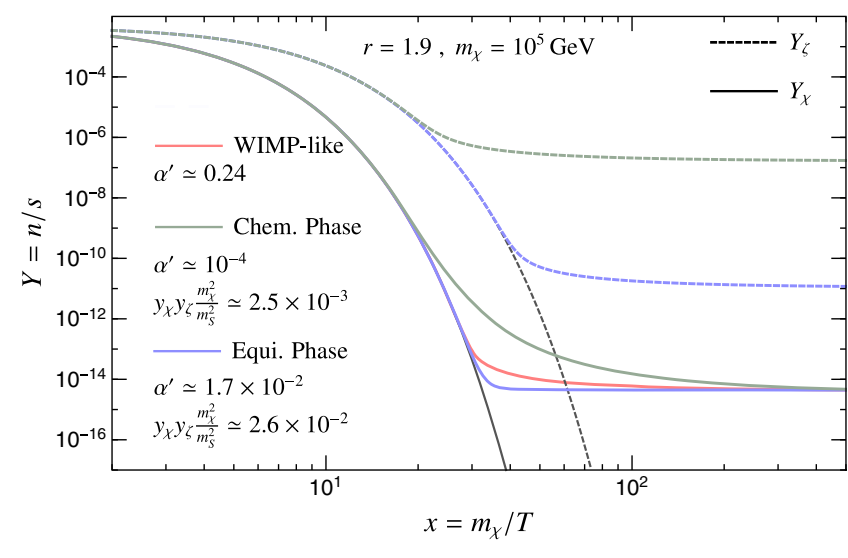

FIG. 2. Thermal evolution of the number density of $\chi$ (solid lines) and $\zeta$ (dashed lines), with $m_{\chi}=1.9 \times m_{\zeta}=10^{6} \mathrm{GeV}$ for interaction rates producing the observed DM relic abundance. Gray lines, equilibrium distributions for $\mu=0$; blue lines, equilibrium phase $(\zeta$ is in chemical equilibrium with the SM bath when $\chi$ freezes out); green lines, chemical phase ( $\zeta$ has departed chemical equilibrium with the SM right before $\chi$ freezes out); red line, WIMP-like ( $\chi$ freezes out like a standard WIMP).

standard WIMP. Parametrizing the annihilation cross section as $\left\langle\sigma_{\chi \chi \rightarrow \mathrm{smsm}} v\right\rangle=\alpha_{\mathrm{wimp}}^{2} / m_{\chi}^{2}$, the condition for zombie collisions to control the abundance is easily determined by comparing Eq. (6) to the analogous equation for the WIMP,

$$
\left[\left(\alpha_{\chi}^{2} m_{\mathrm{pl}}\right)^{r} T_{\mathrm{eq}}\right]^{\frac{1}{1+r}} \gtrsim \alpha_{\mathrm{wimp}}\left(m_{\mathrm{pl}} T_{\mathrm{eq}}\right)^{\frac{1}{2}} .
$$

This equation gives the approximate phase boundary between the WIMP and the equilibrium phase.

Chemical phase-zombies develop chemical potential: It is possible that $\zeta$ freezes out of equilibrium with the SM bath before $\chi$ decouples from $\zeta$. In this case, $\zeta$ has a constant comoving abundance when $\chi$ departs from equilibrium with it. (A nonzero chemical potential of another state also impacts DM freeze-out in Refs. [15,32,42-44].) We show in the Supplemental Material [40] that, in this case,

$$
m_{\chi} \sim\left[\left(\alpha_{\zeta}^{2} m_{\mathrm{pl}}\right)^{r+\Delta} T_{\mathrm{eq}}\right]^{\frac{1}{1+r+\Delta}},
$$

where we have defined $\left\langle\sigma_{\zeta \zeta \rightarrow \mathrm{sm} s m} v\right\rangle \equiv \alpha_{\zeta}^{2} / m_{\chi}^{2}$ and

$$
\Delta \equiv r \frac{x_{\chi}}{x_{\zeta}}+(r-1)\left(\frac{x_{\chi}}{x_{\zeta}}-1\right)>0,
$$

with $x_{\chi}$ and $x_{\zeta}$ defined similar to Eq. (3). We see that the chemical phase leads to even higher DM masses than the equilibrium phase, as can be seen by comparing Eq. (8) to Eq. (6). The thermal evolution for this phase is shown in the green curves in Fig. 2, for the model realization we present below. A chemical potential can affect the calculation of the thermally average cross section [45]. However, since these values are important when the particles are nonrelativistic and therefore in a thermal Maxwell-Boltzmann distribution, the standard thermally average calculation applies.

The crossover between the equilibrium phase and the chemical phase occurs when the rate for $\chi$ to undergo $\chi \zeta^{\dagger} \rightarrow \zeta \zeta$ is approximately the same as the rate for $\zeta$ to annihilate via $\zeta \zeta^{\dagger} \rightarrow \mathrm{sm}$ sm at freeze-out. This is simply the condition $\alpha_{\zeta} \simeq \alpha_{\chi}$.

Additional phases: Since the zombies can freeze out with a large abundance, they can come to dominate the energy of the Universe, leading to an early period of matter domination. If this happens, the $\zeta$ must decay and reheat the radiation bath. The large entropy dump from the decay will change the relic density calculation. We discuss this more in the following section. Finally, if $\zeta$ freezes out when relativistic, the model enters a new phase where the relic abundance of $\chi$ no longer depends on when $\zeta$ freezes out. Additionally, the $\chi$ temperature may not match the SM bath temperature, but the temperatures can be comparable. These two possibilities - nonthermal DM and large dilution via entropy dump — were studied in a similar framework in Ref. [15]. A main goal of this Letter is to demonstrate the possibility of very heavy thermal DM within perturbative unitarity, without modifying early cosmology. For this reason, we leave detailed discussion of these additional phases for future work.

Unitarity and DM decays.-The zombies $\zeta$ are either stable, and themselves constitute a component of dark matter in addition to $\chi$, or are unstable. If the zombies are stable, they must satisfy the unitarity bound, applied to $\zeta \zeta \rightarrow \operatorname{sm~sm}$, and therefore $\chi$ is at most $\mathcal{O}(1)$ heavier by the assumption that $m_{\chi}<3 m_{\zeta}$. If the zombies are unstable, then both $\zeta$ and $\chi$ can have masses that far exceed the unitarity bound. In this case, the abundance of $\zeta$ will exceed the abundance of $\chi$ at freeze-out, but this energy density is removed by decays of $\zeta$. For the remainder of this Letter we focus on unstable zombies.

There are two important phenomenological consequences of the fact that $\zeta$ freezes out with an abundance larger than $\chi$ and subsequently decays. First, if $\zeta$ decays, then $\chi$ is unstable via the process $\chi \rightarrow \zeta \zeta \zeta$, where some or all of the $\zeta$ 's are produced off shell and decay. This leads to potentially strong indirect detection signatures and constraints. Indirect detection constraints on the DM decay lifetime $\tau_{\chi}$, e.g., from the diffuse gamma-ray spectrum, can be as strong as $\tau_{\chi} \gtrsim 10^{27} \mathrm{sec}$ [46-48].

Second, if $\zeta$ freezes out with a large abundance, it can come to dominate the energy of the Universe before it decays away. The large entropy dump that accompanies the decay effectively dilutes $\chi[12,13,16,22,41,49-58]$, allowing for DM masses beyond the WIMP unitarity bound. Additionally, a sufficiently long-lived and abundant $\zeta$ can imply that the Universe was matter dominated during big bang nucleosynthesis (BBN), spoiling the successful predictions of the standard cosmological scenario. 


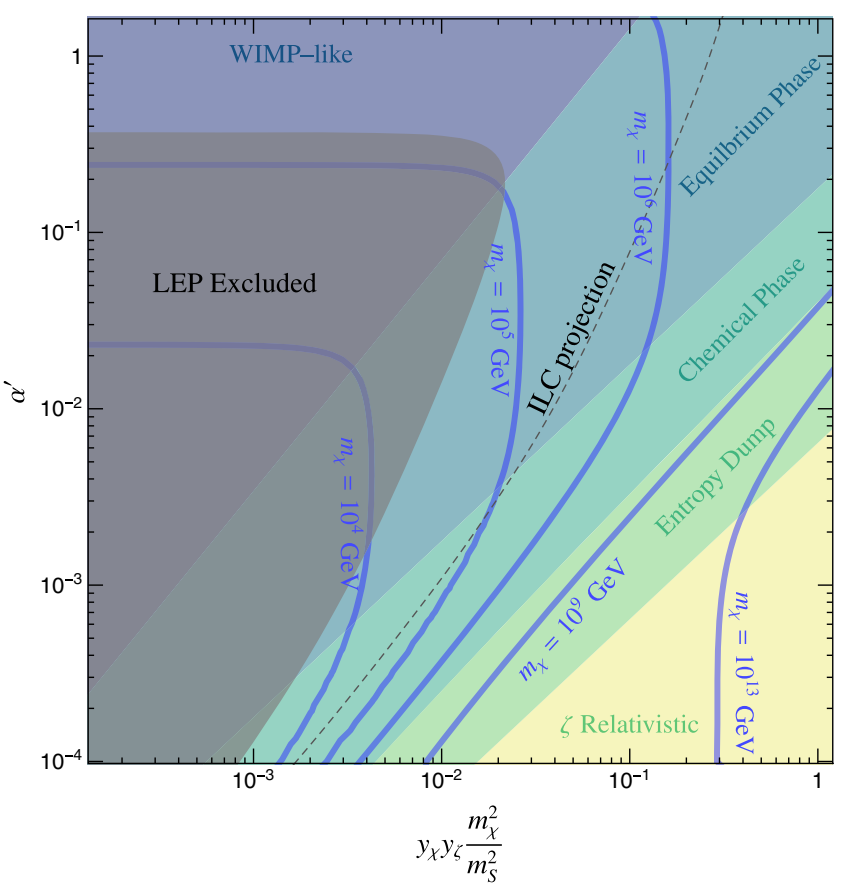

FIG. 3. $\alpha^{\prime}$ vs $y_{\chi} y_{\zeta} m_{\chi}^{2} / m_{S}^{2}$, with contours of constant mass required to match the observed relic abundance. We fix the ratios $m_{\chi}=1.9 m_{\zeta}$ and $m_{Z^{\prime}}=0.1 m_{\chi}$. We take the lifetime of $\chi$ to be $\tau_{\chi}=10^{27}$ sec. Differently colored regions correspond to different phases of the model. See the main text for an explanation of each phase. The shaded gray region is excluded by LEP $Z^{\prime}$ searches, while dashed gray indicates projected international linear collider sensitivity for $Z^{\prime}$ searches.

Example model.-Consider the SM extended with a gauged $\mathrm{U}(1)_{e-\mu}$ lepton number and a dark sector containing fermions $\chi$ and $\zeta$, and a scalar field $S$, with $\mathrm{U}(1)_{e-\mu}$ charges $q_{\zeta}=1, q_{\chi}=3, q_{S}=-2$, and coupling $\alpha^{\prime}$. The most general renormalizable (and parity invariant) Yukawa interactions are

$$
\mathcal{L}_{\text {int }}=y_{\zeta} S \bar{\zeta}^{c} \zeta+y_{\chi} S \bar{\zeta} \chi+y_{e} H \bar{\zeta} L_{e}+y_{\mu} H \bar{\zeta}^{c} L_{\mu}+\text { H.c. },
$$

where $H$ is the Higgs doublet and $L$ is the lepton doublet. We take tree-level kinetic mixing between the new $\mathrm{U}(1)$ and hypercharge to be absent, although it is radiatively generated by $e$ and $\mu$ loops. The Yukawa couplings generate zombie collisions, $\chi \zeta^{\dagger} \rightarrow \zeta \zeta$, while the gauge interactions generate $\zeta \zeta^{\dagger} \rightarrow \mathrm{sm} \mathrm{sm}$, where sm here can be a SM lepton or the $e-\mu$ gauge boson. The Yukawa and gauge interactions also generate $\chi \bar{\chi}$ annihilations, which, if responsible for $\chi$ freeze-out, will lead to a WIMP-like scenario. For other models of leptophilic DM, see, for example, Refs. [59-71].

In Fig. 3 we show curves of constant DM relic abundance $\Omega_{\chi} \simeq 0.27$ [72] and the different phases of freeze-out. Here we take $m_{\chi}=1.9 m_{\zeta}$ and $m_{Z^{\prime}}=0.1 m_{\chi}$, where $Z^{\prime}$ is the massive $\mathrm{U}(1)_{e-\mu}$ gauge boson. Note that, for smaller values of the vector mass $m_{Z^{\prime}}$, the various cross sections will be Sommerfeld enhanced due to the $\mathrm{U}(1)_{e-\mu}$ force, leading to even heavier DM for the same coupling strength [73]. We also take $m_{S} \gtrsim \mathcal{O}($ few $)\left(m_{\chi}+m_{\zeta}\right)$ so that $\chi \bar{\zeta} \rightarrow \zeta \zeta$ is not on the $S$ resonance and can be approximated by a contact interaction. We fix the lifetime of $\chi \rightarrow \zeta H H L L$ to be $\tau_{\chi}=10^{27} \mathrm{sec}$, which is calculated using FeynRules [74] and MadGraph [75].

The results of the thermal evolution of our example model contains different phases, depending on the relative size of the interactions. For large $\mathrm{U}(1)_{e-\mu}$ gauge coupling $\alpha^{\prime}$, the annihilations of $\chi \bar{\chi}$ pairs is very efficient, and the DM behavior is WIMP-like. This is labeled "WIMP-like" in the top left region of Fig. 3, where constant mass lines are horizontal since the relic abundance is insensitive to the Yukawa interactions. As $\alpha^{\prime}$ drops, the $\chi \zeta^{\dagger} \rightarrow \zeta \zeta$ process becomes more efficient at late times. If the gauge coupling is still large enough to maintain chemical equilibrium between the dark and visible sectors until $\chi$ freeze-out, then DM enters the equilibrium phase, corresponding to the vertical lines in the region labeled "equilibrium phase" in Fig. 3. If $\zeta$ freezes out before $\chi$ freezes out, the DM will be in the chemical phase. This phase is further separated into three regions. The first, labeled "chemical phase," signals when $\zeta$ freezes out when nonrelativistic, but never dominates the energy density of the Universe. The second region, labeled "entropy dump," shows where $\zeta$ freezes out nonrelativistically, but dominates the energy density before it decays, leading to a non-negligible dilution of the DM density. The final region, labeled " $\zeta$ relativistic," shows where $\zeta$ freezes out while still relativistic. Unitarity bounds correspond roughly to $\alpha^{\prime} \sim 1, y_{\chi} y_{\zeta} m_{\chi}^{2} / m_{S}^{2} \sim 1$, for $m_{\chi} / m_{S} \sim 10^{-1}$. Note that, for much heavier $m_{S}$, the unitarity bound will be stricter unless we allow for strong (nonperturbative) couplings in the UV theory.

Signatures and constraints. -We now discuss the generic phenomenological signals of the mechanism and signatures and constraints of the leptophilic model presented above. Since the focus of this Letter is on heavy DM, we discuss only parameter regions that correspond to $m_{\chi} \gtrsim 100 \mathrm{GeV}$.

The mechanism generically predicts an indirect detection signal from dark sector decays. The $\zeta$ particles must decay before $\mathrm{BBN}$ to not obstruct light element formation [76,77]. On the other hand, a decay of $\zeta$ induces a decay of $\chi$ through the interaction $\chi^{\dagger} \zeta \zeta \zeta$. Late-time $\chi$ decays may produce ultrahigh energy cosmic rays (UHECRs), detectable by diffuse gamma-ray satellites [78], high energy neutrino experiments [79], and in dedicated UHECR observatories [80]. Combined with the BBN bound, the decay rate of $\zeta$ must reside in the window $H_{\mathrm{BBN}} \lesssim \Gamma_{\zeta} \lesssim \Gamma_{\max }$, where $\Gamma_{\max }$ is the value of $\Gamma_{\zeta}$ such that the lifetime of $\chi$ is within indirect detection bounds. 
This requires the Yukawa couplings $y_{e}$ and $y_{\mu}$ to be as small as $10^{-13}$ within the chemical phase. In Fig. 3 we fix $\Gamma_{\chi}^{-1}=\left(10^{27} \mathrm{sec}\right)^{-1}$. For the plotted parameter ranges, $\Gamma_{\max }>H_{\mathrm{BBN}}$ everywhere, showing the possibility of a large indirect detection signal for all masses.

The portal between the sectors leads to collider signatures. In Fig. 3, we show the constraints from the measurement of the differential cross section of lepton pairs at large electron-positron (LEP) collider [81], which bounds $m_{Z^{\prime}} / \sqrt{\alpha^{\prime}}>25 \mathrm{TeV}$, as well as future projections for the international linear collider with reach $m_{Z^{\prime}} / \sqrt{\alpha^{\prime}}>$ $200 \mathrm{TeV}$ [66]. Dark production at the LHC for our model is suppressed because there is no tree-level interaction with quarks. However, due to the long-lived nature of $\zeta$, the dark sector may be discoverable in experiments designed to look for long-lived particles at the LHC, such as AL3X [82], CODEX-b [83], FASER [84], and MATHUSLA [85]. Finally, if a gauged $\mathrm{U}(1)_{B-L}$ is considered instead of the leptophilic model, the freeze-out would remain unchanged, but there would be stronger collider signatures, which we leave for future work.

As mentioned above, we have set the tree-level gauge kinetic mixing to zero. DM-proton scattering is generated at one loop by the electron and muon with cross section given by [70,86-88]

$$
\sigma_{p}=\frac{64 \mu_{\chi p}^{2}}{\pi m_{Z^{\prime}}^{4}} \alpha_{\mathrm{em}}^{2} \alpha^{\prime 2} \log ^{2}\left(\frac{m_{e}}{m_{\mu}}\right)
$$

where $\mu_{\chi p}$ is the reduced DM-proton mass. Although this strength of interaction might seem relevant for direct detection experiments, we find that current nucleon recoil direct detection constraints from XENON1T [89] do not outperform LEP $Z^{\prime}$ searches. In the lower $\chi$ mass end of our model $\left(m_{\chi} \lesssim \mathrm{GeV}\right)$, electron recoil experiments might be relevant and one would have to take into account the effect of shielding by the Earth [90], which in our case is dominated by a loop induced interaction with protons.

We would like to thank Asher Berlin, Timothy Cohen, Timon Emken, Rouven Essig, Michael Geller, Roni Harnik, Yonit Hochberg, Hyungjin Kim, Gordan Krnjaic, Sam McDermott, Mukul Sholapurkar, and Tomer Volansky for useful discussions. The work of E. D. K. is supported by the Zuckerman STEM Leadership Program. The work of E. K. and E. D. K. is supported by the Israel Science Foundation (Grant No. 1111/17), and by the I-CORE Program of the Planning Budgeting Committee (Grant No. 1937/12). The work of E. K. is further supported by the Binational Science Foundation (Grant No. 2016153). N. J. O. is grateful to the Azrieli Foundation for the support of an Azrieli Fellowship. N. L. would like to thank the Milner Foundation for the support of a Milner Fellowship. J. T. R. is supported by NSF CAREER Grant No. PHY1554858 and NSF Grant No. PHY-1915409. E. D. K.,
E. K., and N. J. O. would like to acknowledge the GGI Institute for Theoretical Physics for enabling them to complete a significant portion of this work. J. T. R. acknowledges hospitality from the Aspen Center for Physics, which is supported by the NSF Grant No. PHY-1607611.

*ericdavidkramer@gmail.com

†eric.kuflik@mail.huji.ac.il

"noam@mail.tau.ac.il

${ }^{\S}$ Nadav.Out@gmail.com

ruderman@nyu.edu

[1] K. Griest and M. Kamionkowski, Phys. Rev. Lett. 64, 615 (1990).

[2] L. Hui and E. D. Stewart, Phys. Rev. D 60, 023518 (1999).

[3] E. W. Kolb, D. J. H. Chung, and A. Riotto, AIP Conf. Proc. 484, 91 (1999).

[4] D. J. H. Chung, E. W. Kolb, and A. Riotto, Phys. Rev. D 60, 063504 (1999).

[5] D. J. H. Chung, P. Crotty, E. W. Kolb, and A. Riotto, Phys. Rev. D 64, 043503 (2001).

[6] J. L. Feng, H. Tu, and H.-B. Yu, J. Cosmol. Astropart. Phys. 10 (2008) 043.

[7] K. Harigaya, M. Kawasaki, K. Mukaida, and M. Yamada, Phys. Rev. D 89, 083532 (2014).

[8] H. Davoudiasl, D. Hooper, and S. D. McDermott, Phys. Rev. Lett. 116, 031303 (2016).

[9] L. Randall, J. Scholtz, and J. Unwin, J. High Energy Phys. 03 (2016) 011.

[10] P. S. B. Dev, R. N. Mohapatra, and Y. Zhang, J. High Energy Phys. 11 (2016) 077.

[11] K. Harigaya, T. Lin, and H. K. Lou, J. High Energy Phys. 09 (2016) 014.

[12] A. Berlin, D. Hooper, and G. Krnjaic, Phys. Lett. B 760, 106 (2016).

[13] A. Berlin, D. Hooper, and G. Krnjaic, Phys. Rev. D 94, 095019 (2016).

[14] J. Bramante and J. Unwin, J. High Energy Phys. 02 (2017) 119.

[15] A. Berlin, Phys. Rev. Lett. 119, 121801 (2017).

[16] S. Hamdan and J. Unwin, Mod. Phys. Lett. A 33, 1850181 (2018).

[17] M. Cirelli, Y. Gouttenoire, K. Petraki, and F. Sala, J. Cosmol. Astropart. Phys. 02 (2019) 014.

[18] E. Babichev, D. Gorbunov, and S. Ramazanov, Phys. Lett. B 794, 69 (2019).

[19] S. Hashiba and J. Yokoyama, Phys. Rev. D 99, 043008 (2019).

[20] D. Hooper, G. Krnjaic, and S. D. McDermott, J. High Energy Phys. 08 (2019) 001.

[21] H. Davoudiasl and G. Mohlabeng, J. High Energy Phys. 04 (2020) 177.

[22] P. Chanda, S. Hamdan, and J. Unwin, J. Cosmol. Astropart. Phys. 01 (2020) 034.

[23] D. Chway, T. H. Jung, and C. S. Shin, Phys. Rev. D 101, 095019 (2020).

[24] K. Harigaya, M. Ibe, K. Kaneta, W. Nakano, and M. Suzuki, J. High Energy Phys. 08 (2016) 151. 
[25] J. Smirnov and J. F. Beacom, Phys. Rev. D 100, 043029 (2019).

[26] R. Contino, A. Mitridate, A. Podo, and M. Redi, J. High Energy Phys. 02 (2019) 187.

[27] C. Gross, A. Mitridate, M. Redi, J. Smirnov, and A. Strumia, Phys. Rev. D 99, 016024 (2019).

[28] M. Geller, S. Iwamoto, G. Lee, Y. Shadmi, and O. Telem, J. High Energy Phys. 06 (2018) 135.

[29] F. D’Eramo and J. Thaler, J. High Energy Phys. 06 (2010) 109.

[30] Y. Hochberg, E. Kuflik, T. Volansky, and J. G. Wacker, Phys. Rev. Lett. 113, 171301 (2014).

[31] E. Kuflik, M. Perelstein, Nicolas Rey-Le Lorier, and Y.-D. Tsai, Phys. Rev. Lett. 116, 221302 (2016).

[32] J. M. Cline, H. Liu, T. R. Slatyer, and W. Xue, Phys. Rev. D 96, 083521 (2017).

[33] R. T. D’Agnolo, D. Pappadopulo, and J. T. Ruderman, Phys. Rev. Lett. 119, 061102 (2017).

[34] J. Smirnov and J. F. Beacom, Phys. Rev. Lett. 125, 131301 (2020).

[35] H. Kim and E. Kuflik, Phys. Rev. Lett. 123, 191801 (2019).

[36] K. Griest and D. Seckel, Phys. Rev. D 43, 3191 (1991).

[37] R. T. D'Agnolo and J. T. Ruderman, Phys. Rev. Lett. 115, 061301 (2015).

[38] M. Garny, J. Heisig, B. Lulf, and S. Vogl, Phys. Rev. D 96, 103521 (2017).

[39] R. T. D’Agnolo, D. Pappadopulo, J. T. Ruderman, and P.-J. Wang, Phys. Rev. Lett. 124, 151801 (2020).

[40] See Supplemental Material at http://link.aps.org/supplemental/ 10.1103/PhysRevLett.126.081802 for details and derivations.

[41] E. W. Kolb and M. S. Turner, Front. Phys. 69, 1 (1990).

[42] P. Bandyopadhyay, E. J. Chun, and J.-C. Park, J. High Energy Phys. 06 (2011) 129.

[43] M. Farina, D. Pappadopulo, J. T. Ruderman, and G. Trevisan, J. High Energy Phys. 12 (2016) 039.

[44] J. A. Dror, E. Kuflik, and W. H. Ng, Phys. Rev. Lett. 117, 211801 (2016).

[45] T. Bringmann, P. F. Depta, M. Hufnagel, and K. SchmidtHoberg, arXiv:2007.03696.

[46] M. Cirelli, E. Moulin, P. Panci, P. D. Serpico, and A. Viana, Phys. Rev. D 86, 083506 (2012).

[47] R. Essig, E. Kuflik, S. D. McDermott, T. Volansky, and K. M. Zurek, J. High Energy Phys. 11 (2013) 193.

[48] C. Blanco and D. Hooper, J. Cosmol. Astropart. Phys. 03 (2019) 019.

[49] R. J. Scherrer and M. S. Turner, Astrophys. J. 331, 19 (1988).

[50] K. Jedamzik, Phys. Rev. Lett. 84, 3248 (2000).

[51] E. A. Baltz and H. Murayama, J. High Energy Phys. 05 (2003) 067.

[52] T. Asaka, M. Shaposhnikov, and A. Kusenko, Phys. Lett. B 638, 401 (2006).

[53] A. V. Patwardhan, G. M. Fuller, C. T. Kishimoto, and A. Kusenko, Phys. Rev. D 92, 103509 (2015).

[54] T. Tenkanen and V. Vaskonen, Phys. Rev. D 94, 083516 (2016).

[55] E. Hardy, J. High Energy Phys. 06 (2018) 043.

[56] N. Bernal, C. Cosme, and T. Tenkanen, Eur. Phys. J. C 79, 99 (2019).

[57] R. Allahverdi et al., arXiv:2006.16182.
[58] M. Kawasaki, K. Kohri, T. Moroi, K. Murai, and H. Murayama, arXiv:2006.14803.

[59] P. J. Fox and E. Poppitz, Phys. Rev. D 79, 083528 (2009).

[60] W. Chao, Phys. Lett. B 695, 157 (2011).

[61] S. Chang, R. Edezhath, J. Hutchinson, and M. Luty, Phys. Rev. D 90, 015011 (2014).

[62] Y. Bai and J. Berger, J. High Energy Phys. 08 (2014) 153.

[63] N. F. Bell, Y. Cai, R. K. Leane, and A. D. Medina, Phys. Rev. D 90, 035027 (2014).

[64] P. Schwaller, T. M. P. Tait, and R. Vega-Morales, Phys. Rev. D 88, 035001 (2013).

[65] J. Kile, A. Kobach, and A. Soni, Phys. Lett. B 744, 330 (2015).

[66] A. Freitas and S. Westhoff, J. High Energy Phys. 10 (2014) 116.

[67] B. Fornal, Y. Shirman, T. M. P. Tait, and J. R. West, Phys. Rev. D 96, 035001 (2017).

[68] S. Dutta, D. Sachdeva, and B. Rawat, Eur. Phys. J. C 77, 639 (2017).

[69] E. Madge and P. Schwaller, J. High Energy Phys. 02 (2019) 048.

[70] G. H. Duan, X.-G. He, L. Wu, and J. M. Yang, Eur. Phys. J. C 78, 323 (2018).

[71] H. Banerjee and S. Roy, Phys. Rev. D 99, 035035 (2019).

[72] N. Aghanim et al. (Planck Collaboration), arXiv:1807.06209.

[73] N. Arkani-Hamed, D. P. Finkbeiner, T. R. Slatyer, and N. Weiner, Phys. Rev. D 79, 015014 (2009).

[74] A. Alloul, N. D. Christensen, C. Degrande, C. Duhr, and B. Fuks, Comput. Phys. Commun. 185, 2250 (2014).

[75] J. Alwall, R. Frederix, S. Frixione, V. Hirschi, F. Maltoni, O. Mattelaer, H. S. Shao, T. Stelzer, P. Torrielli, and M. Zaro, J. High Energy Phys. 07 (2014) 079.

[76] J. R. Ellis, D. V. Nanopoulos, and S. Sarkar, Nucl. Phys. B259, 175 (1985).

[77] M. Kawasaki, K. Kohri, T. Moroi, and Y. Takaesu, Phys. Rev. D 97, 023502 (2018).

[78] M. Ackermann et al. (Fermi-LAT Collaboration), Astrophys. J. 799, 86 (2015).

[79] R. Abbasi et al. (IceCube Collaboration), Phys. Rev. D 83, 092003 (2011); 84, 079902(E) (2011).

[80] P. Abreu et al. (Pierre Auger Collaboration), Phys. Rev. D 84, 122005 (2011).

[81] S. Schael et al. (ALEPH, DELPHI, L3, OPAL, LEP Electroweak Collaborations), Phys. Rep. 532, 119 (2013).

[82] V. V. Gligorov, S. Knapen, B. Nachman, M. Papucci, and D. J. Robinson, Phys. Rev. D 99, 015023 (2019).

[83] V. V. Gligorov, S. Knapen, M. Papucci, and D. J. Robinson, Phys. Rev. D 97, 015023 (2018).

[84] J. L. Feng, I. Galon, F. Kling, and S. Trojanowski, Phys. Rev. D 97, 035001 (2018).

[85] D. Curtin et al., Rep. Prog. Phys. 82, 116201 (2019).

[86] B. Holdom, Phys. Lett. 166B, 196 (1986).

[87] G. Arcadi, M. D. Campos, M. Lindner, A. Masiero, and F. S. Queiroz, Phys. Rev. D 97, 043009 (2018).

[88] C. Blanco, M. Escudero, D. Hooper, and S. J. Witte, J. Cosmol. Astropart. Phys. 11 (2019) 024.

[89] E. Aprile et al. (XENON Collaboration), Phys. Rev. Lett. 119, 181301 (2017).

[90] T. Emken, R. Essig, C. Kouvaris, and M. Sholapurkar, J. Cosmol. Astropart. Phys. 09 (2019) 070. 